\title{
Towards Quantum Experiments with Human Eyes Detectors Based on Cloning via Stimulated Emission?
}

\author{
Francesco De Martini \\ Dipartimento di Fisica, Sapienza Universitá di Roma, Roma, 00185 Italy and \\ Accademia Nazionale dei Lincei, via della Lungara 10, I-00165 Roma, Italy
}

\begin{abstract}
We believe that a recent theoretical work published in Physical Review Letters (103, 113601, 2009) by Sekatsky, Brunner, Branciard, Gisin, Simon, albeit appealing at fist sight, is highly questionable. Furthermore, the criticism raised by these Authors against a real experiment on Micro - Macro entanglement recently published in Physical Review Letters $(100,253601,2008)$ is found misleading and misses its target.

PACS numbers:
\end{abstract}

We believe that the work by P.Sekatsky, N.Brunner, C. Branciard, N. Gisin and C.Simon is highly questionable [1]. The first seed of perplexity is elicited by the title of the paper, the same as the one of the present article (apart from the question mark). For the eye of a human observer, as well as any other human sensory organ, just cannot be adopted as a valid "measurement apparatus" within any experiment involving a quantum mechanical process. As stated many times by Niels Bohr, this apparatus must be a "classical" one, i.e. whose behavior follows reliably the well established, deterministic laws of classical physics [2]. This is vividly expressed by the prose of J.A.Wheeler [3]: "A phenomenon is not yet a phenomenon until is a registered phenomenon i.e. brought to a close by an irreversible act of amplification". The measurement apparatus must be composed by three devices: (a) A detector, e.g. a photocathode or a grain of silver bromide etc, that realizes the reduction of the quantum wavefunction, (b) A classical amplifier (c) A registering unit, or a memory that records the outcome of the measurement, i.e. a real number. Indeed the last device is a utterly necessary item in order "to bring to a close" the measurement and then to establish "the phenomenon". We remind here that the "memory erasure" (or the "register re-setting") was the necessary conceptual step taken by Charles Bennett in order to resolve the famous "Maxwell demon Paradox" [4] In short, a measurement is far more than a mere perception, i.e. a solipsistic process. Any measurement outcome must be available to the "observer" as well as to an independent scientific community by which it can be promoted to the level of "datum" ready to be adopted by a scientific theory. Quite obviously, the eye detection does not comply with these requirements for several reasons. First, the "recording device" is not in front of but rather literally in the head of the observer. Then, to say the least, the measurement outcomes cannot be independently addressed by other observers. Second, we couldn't imagine which "mental pointer" or mental counter or mental scaling algorithm could be adopted in order to transform, in a reliable and reproducible way, the level of the synaptic electric field into one amongst a set of orthogonal outcomes expressing the detected light intensity, i.e. the only "observable" accessible to the eye. In facts the "pointer" sitting somewhere within the brain of the observer should be able to single out a definite orthogonal outcome (a), say: $\mathrm{a}=3$, rather than: $\mathrm{a}=2$, or $\mathrm{a}=$ 4. Third, while the role of the retina and the $\mathrm{Na}$ - ion excitation dynamics of the optical nerve may be taken as rather well understood, the complex synaptic trasmission to the "reentrant" talamo - cortical system of the brain [5] and the consequent establisment of the various levels of memory [6] are obscure and are today the subject of frontier research within the domain of the advanced neurosciences. For the present purpose we only know for sure some phenomenological properties of the brain. For instance, that the optical nerve amplification is highly nonlinear and that the overall visual efficiency is easily saturated. Furthermore, some drugs as alchool (contained in wine) and betacarotene (contained in carrots or tomatoes) not to speak of other more dangerous drugs can have large and opposite effects on the parameters of visual speed, nonlinearity and efficiency. Indeed too many scarcely controllable things can happen simultaneously within our head in any moment: perceptions, emotions, desires, phantasies, rational and irrational thoughts: all that is part of the rich realm of "consciousness". Then, while we largely sympatize with the open mindness revealed by the work done in the domain of measurement related consciousness by scientists as Von Neumann [7], Pauli[8], Wigner [9], Stapp [10] and others, we can't restrain from manifesting our opposition when dealing with serious real experiments and theories. In summary, we believe that today, and foreseeably for a very long time in the future, the human eye detection should be thought of as a largely useless "epistemic mess" when related to the argument of quantum measurement.

The bravery of P.Sekatsky et al. with their eye detection proposal is further revealed by the gedanken experiment considered in [1] and dealing with a nonlocality test 


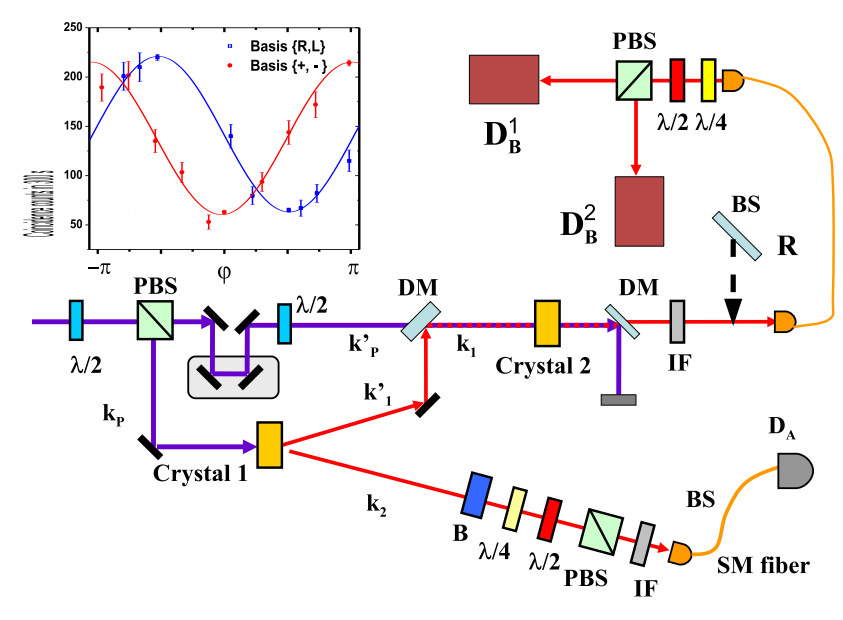

FIG. 1: Layout of the Micro-Macro experiment published in Phys.Rev. Lett. 100, 253601 (2008). At the output of the QIOPA Amplifier an optional beam-splitter with reflectivity $\mathrm{R}$ simulates the photon - loss in the detection process. In order to determine the visibilities $V_{2}$ and $V_{3}$, the couples of experimental points corresponding to the maxima and minima of the fringing patterns obtained in correspondence with two different measurement bases have been determined by higher statistics and exhibit a smaller error flag.

made on a micro-macro photon system by the violation of a Bell inequality. We may describe the proposed experiment on the basis of their Figure 3, as follows. A standard laser system generates by Spontaneous Parametric Down Conversion (SPDC) a polarization singlet couple of photons that are sent to two spacelike distant stations, Alice and Bob. The single photon received by Alice is measaured by a standard Optical Stern-Gerlach apparatus $\left(O S G_{A}\right)$ consisting of a couple of single-photon detectors coupled to the two output modes of a Polarizing Beam Splitter $P B S_{A}$. As usual in these experiments, the $O S G_{A}$ is "rotated" of an angle $\Phi_{A}$ (All generalized "rotations" considered in the present letter could possibly imply changes of the state of photon polarization, e.g. from "linear" to " circular" etc.) Likewise, the single photon send towards Bob, is amplified by some unspecified device. The $N$ photons generated by the amplifier are then measured by another Optical Stern-Gerlach system $\left(O S G_{B}\right)$ consisting of a $P B S_{B}$ "rotated" by an angle $\Phi_{B}$. Needless to say, the $\left(O S G_{B}\right)$ is completed by two naked human eyes, accurately drawn in the Figures 1 and 3 of [1], each one staring in one of the output modes of the $P B S_{B}$. This proposed gedanken experiment reproduces almost exactly the real experiment previously carried out by [12] where the amplifier is a Quantum Injected Optical Parametric Amplifier (QI-OPA) generating $N \simeq 10^{5}$ output photons [11] and the human eyes are replaced, perhaps more reasonably, by two detection apparata $D_{B}^{1}, D_{B}^{2}$ each involving a linear photomultiplier .
The experimental layout of our real experiment is shown in Figure 1, above.

The gedanken experiment planned by Sebatsky et al. raises further obvious questions. For instance, the two naked eyes, in order to be able to measure different signals, cannot belong to the same person because of the physiological fusion of the related perceptions due to the inextricable and incontrollable interconnections between the optical nerves reaching the left and right sectors of the same brain. The eyes must then belong to two different persons (two students ?). At that point, since the Bell inequality experiments with many photons cannot imply simple yes/no responses but require the registering of the actual level of the synaptic signals, we are again confronted with slippery unanswerable questions about mental pointers, mental signal processing, saturation, linearity, neural connections, betacarotene and alchool, mutual calibration and stability, consciousness etc.

At last, let's stop pondering on the bizarre naked eye detection idea and do consider the detailed micro-macro Bell inequality theory also reported in [1]. There a measurement loophole is devised in physical situations implying the calculation of the joint correlation parameters between apparata $\left(O S G_{A}\right)$ and $\left(O S G_{B}\right)$ tuned on different measurement bases, i.e. when the relative angular settings of the corresponding measurement apparata differ from zero: $\Delta \Phi \equiv\left|\Phi_{A}-\Phi_{B}\right| \neq 0$. Indeed, this is a typical situation realized in all Bell inequality experiments. We don't disagree on several results of the theoretical analysis by 1] but we also want to stress that these ones are quite incorrectly applied to the real experiment reported in 12. In other words, the criticism to our work by Sebatsky et al, presumably the true motivation of work [1], is misleading as it misses completely the point. For the following reasons:

(A) The work [12] is not a Bell inequality experiment and then no correlations between different measurement bases are measured or calculated within the same experiment.. The work [12] merely consists of two totally independent and uncorrelated experiments aimed at the evaluation of two different and uncorrelated quantities, i.e. the "visibilities" $V_{2}$ and $V_{3}$ of the two different and uncorrelated fringing patterns shown in Figure 1, above. (The other "visibility" was found: $V_{1} \simeq 0$ ). These patterns, drawn as function of $\Phi_{B}$, represent the jointly correlated detection probabilities when a fixed measurement basis of $\left(O S G_{A}\right)$ is chosen to be either $\{R, L\}$ or $\{+,-\}$, respectively. Consider for instance the measurement of $V_{2}$, i.e. the visibility of the fringe pattern determined by the fixed basis $\{R, L\}$ set at the Alice's site. As it is well known $V_{2}$ is determined by only two points, the maximum and the minimum of the pattern, i.e. exactly the points corresponding to the conditions: $\Phi_{A}=\Phi_{B}$, or: $\Delta \Phi=0$.In other words, the two data used to evaluate $V_{2}$ are obtained by measuring the joint detection probabili- 


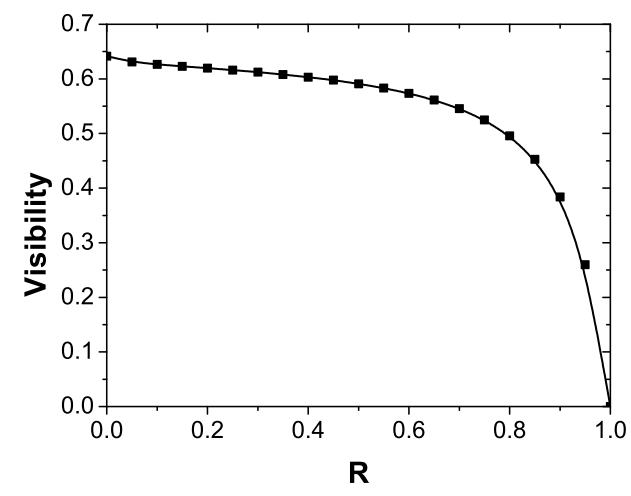

FIG. 2: Complete computer simulation of the experiment [12] showing the decrease of the visibility $V_{2}$ due to the reduction of micro - macro entanglement for increasing $R$, i.e. the amount of photon loss. .

ties in the conditions in which the micro-qubit at Alice's site and the macro-qubit at Bob's site are mutually parallel or anti - parallel spin vectors i.e. both belonging to the same $\{R, L\}$ basis on the corresponding, equally oriented Poincaré spheres. The same condition: $\Phi_{A}=$ $\Phi_{B}$, or: $\Delta \Phi=0$ is realized within the measurement of $V_{3}$ where again the common measurement basis $\{+,-\}$ is realized for both the Alice's and Bob's apparata. Then, because of the common condition: $\Delta \Phi=0$ affecting both measurements of $V_{2}$ and $V_{3}$, the "loophole" devised by Sekatsky et al. is not applicable to our experiment.

(B) Symmetry considerations based on the rotational invariance of the overall micro-macro singlet photon pair expressed by Equation 1 in [12], and of the phasecovariant and information preserving properties of the of the adopted QI-OPA, lead to conclude that the two $V_{2}$ and $V_{3}$ experiments are really identical, in the sense that the micro and macro states adopted in both cases, albeit formally different, are in fact obtained by relabelling for different polarizations the Fock state components of these micro and macro-states. In facts, the experimental outcomes $V_{2}, V_{3}$ of the two corresponding experiments have been found equal by [12], within the statistical errors.

(C) As presumed by Sekatsky et al, photon losses are indeed present in the multi-photon (Bob) side of experiment [12], mostly due to the reduced quantum efficiency $Q E<1$ of the photomultipliers. In any case the effect of losses is a "local" one and may be modelled, as shown above in Figure 1, by a Beam Splitter (BS) with a transmission $T \equiv(1-R)$ placed right at the output of the QI-OPA apparatus. The result of a complete computer simulation of the experiment [12] by adopting the real experimental parameters and by assuming the fixed measurement basis $\{R, L\}$, is shown in Figure 2 . There the "visibility" $V_{2}$, reported as function of $R$. is found to be a decreasing function of of the amount of photon losses. This result is expected since, being the micro-macro entanglement distributed between all photons emitted by QI-OPA, any photon loss entails a reduction of the amount of entanglement detected on the remaining photons. Furthermore, this behavior agrees with a nice "entanglement criterion" expressed in a paper by Eisenberg et al [13] that can be expressed as follows: "any local transformation cannot enhance the level of entanglement". A photon loss is indeed a local transformation, by definition. The work by Eisenberg et al. 13] also dealt with experimental multiphoton etanglement detection with $Q E<1[17]$..

In spite of the entanglement reduction due to the measurement losses, the "visibility inequality" $\left|V_{1}+V_{2}+V_{3}\right| \leq 1$ was violated in the experiment [12]. This a fortiori demonstrates the nonseparability of our Micro - Macro system.

In summary, all previous considerations fully support our claim asserting that the work 12], taken together with previous works by our Laboratory [11] 14] indeed consists of the first exact realization of the Macroscopic Quantum Superposition, i.e. complying exactly with the original definition given by Schrödinger in 1935 [15]. The value of this discovery is further enhanced by the large resilience to decoherence shown by our system, which involves as many as $N \simeq 10^{5}$ particles [16]. The robustness against any kind of noise makes our system apt to the investigation on several so far inaccessible fundamental issues of quantum mechanics close to the elusive "quantum - classical boundary".

We conclude by stressing our deep appreciation for the continuous interest in our work by P.Sekatsky, N.Brunner, C.Branciard, N. Gisin and C. Simon.

[1] P. Sekatsky et al., Phys. Rev. Lett. 103, 113601 (2009).

[2] N. Bohr, Atomic Physics and Human Knowledge (Ox Bow Press, Woodbridge, Conn. 1963).

[3] J.A. Wheeler, Law without Law in: Quantum Theory and Measurement, J.A. Wheeler and W.H. Zurek eds. (Princeton University Press, 1983).

[4] C.H.Bennett, Int. J. Theor .Phys. 21, 905 (1982); Sci. Am. 257, 108 (1987).

[5] G. M. Edelman, Mindful Brain: Cortical Organization and the Group - selective theory of higher Brains (MIT Press, Cambridge 1978).

[6] E. R. Kandel, In Search of Memory (Norton, New York 2006).

[7] J.Von Neumann, Matematischen Grundlangen der Quantenmechanik (Springer, Berlin 1932)

[8] H. Atmanspacher, H.Primas, Recasting Reality (Springer, Berlin 2009).

[9] E.P. Wigner, Symmetries and Reflections (University of Indiana, Bloomington 1967) 
[10] H.P: Stapp, Mind, Matter and Quantum Mechanics (Springer, Berlin 1993).

[11] F. De Martini, Phys. Rev. Lett. 81, 2842 (1998); Phys. Lett. A 250, 15 (1998).

[12] F. De Martini, F.Sciarrino, and C. Vitelli, Phys. Rev. Lett. 100, 253601 (2008).

[13] H. Eisenberg, G. Khoury, A. Durkin, C. Simon, and D. Bouwmeester, Phys. Rev. Lett. 93, 193901 (2004).

[14] F. De Martini, F. Sciarrino, and V. Secondi, Phys. Rev. Lett. 95, 240401 (2005); F. De Martini, and F. Sciarrino, Progr. Quantum Electr. 29, 165 (2005); F. De Martini, and F. Sciarrino, Journal of Physics A: Math. Theor. 40, 2977 (2007).

[15] E. Schrödinger, Naturwissenshaften 23, 807 (1935).

[16] F. De Martini, F. Sciarrino and N. Spagnolo, Phys. Rev. Lett. 103, 100501 (2009).

[17] Since Christoph Simon co-authored both papers [13] and 1] he may perhaps explain why the "loophole" problems should be applicable to work [12] and not to [13] and why the "visibility inequality" and the "entanglement criterion" should be applicable to work [13] and not to [12] 